\title{
Critical Height of the Torus Instability in Two-ribbon Solar Flares
}

\author{
Dong Wang ${ }^{1,2}$, Rui Liu ${ }^{1}$, Yuming Wang ${ }^{1}$, Kai Liu ${ }^{1}$, Jun Chen ${ }^{1}$, Jiajia Liu ${ }^{1}$, Zhenjun Zhou ${ }^{1}$, and Min Zhang ${ }^{2}$ \\ ${ }^{1}$ CAS Key Laboratory of Geospace Environment, Department of Geophysics and Planetary Sciences, University of Science and Technology of China, \\ Hefei, Anhui 230026, China; rliu@ustc.edu.cn \\ ${ }^{2}$ Department of Mathematics and Physics, Anhui Jianzhu University, Hefei 230601, China \\ Received 2017 April 14; revised 2017 June 9; accepted 2017 June 9; published 2017 June 27
}

\begin{abstract}
We studied the background field for 60 two-ribbon flares of M-and-above classes during 2011-2015. These flares are categorized into two groups, i.e., eruptive and confined flares, based on whether a flare is associated with a coronal mass ejection or not. The background field of source active regions is approximated by a potential field extrapolated from the $B_{z}$ component of vector magnetograms provided by the Helioseismic and Magnetic Imager. We calculated the decay index $n$ of the background field above the flaring polarity inversion line, and defined a critical height $h_{\text {crit }}$ corresponding to the theoretical threshold $\left(n_{\text {crit }}=1.5\right)$ of the torus instability. We found that $h_{\text {crit }}$ is approximately half of the distance between the centroids of opposite polarities in active regions and that the distribution of $h_{\text {crit }}$ is bimodal: it is significantly higher for confined flares than for eruptive ones. The decay index increases monotonously with increasing height for $86 \%$ (84\%) of the eruptive (confined) flares but displays a saddle-like profile for the rest, $14 \%(16 \%)$, which are found exclusively in active regions of multipolar field configuration. Moreover, $n$ at the saddle bottom is significantly smaller in confined flares than that in eruptive ones. These results highlight the critical role of background field in regulating the eruptive behavior of two-ribbon flares.
\end{abstract}

Key words: instabilities - Sun: coronal mass ejections (CMEs) - Sun: flares - Sun: magnetic fields

\section{Introduction}

Solar flares and coronal mass ejections (CMEs) are among the most energetic phenomena in the solar system. They are often associated with each other and hence believed to be governed by the same physical process (Zhang et al. 2001, 2004; Priest \& Forbes 2002; Harrison 2003). In the "standard" picture (Shibata 1998), a positive feedback is established between the slow rising of a magnetic flux rope and magnetic reconnection underneath; as a result, the flux rope erupts into interplanetary space as a CME, and the reconnection is mapped to the solar surface as two-flare ribbons. However, some flares may exhibit circular-shaped (e.g., Liu et al. 2015) or X-shaped ribbons (e.g., Liu et al. 2016b), and not all flares are associated with CMEs (Yashiro et al. 2005). Conventionally, flares are categorized as eruptive flares (with CME association) and confined flares (without CME association). Wang \& Zhang (2007) suggested that eruptive flares differ from confined ones in both the energy release location and the ratio between magnetic flux in the low $\left(<1.1 R_{\odot}\right)$ and high $\left(>1.1 R_{\odot}\right)$ corona. Relevant to the ratio is the torus instability, which has been recognized as a pertinent MHD instability underlying solar eruptions from both theoretical (van Tend \& Kuperus 1978; Kliem \& Török 2006; Aulanier et al. 2010) and observational perspectives (e.g., Török \& Kliem 2005; Liu 2008; Cheng et al. 2011; Xu et al. 2012; Zuccarello et al. 2014; Sun et al. 2015; Liu et al. 2016). The torus instability occurs when the external field above the flux rope decreases too rapidly with increasing height, which is quantified by the decay index $n=-d \ln$ $B / d \ln h$. The threshold value of the instability $n_{\text {crit }}$ is derived to be 1.5 for a toroidal current channel (Kliem \& Török 2006), while for a very flat, nearly two-dimensional current channel, $n_{\text {crit }} \gtrsim 1$ (Démoulin \& Aulanier 2010). On the other hand, some numerical studies (e.g., Fan \& Gibson 2007; Kliem et al. 2013; Zuccarello et al. 2016) and laboratory experiments (Myers et al. 2015, 2016, 2017) found that $n_{\text {crit }}$ is in the range [1.4-2.0].
Before the above discrepancy is resolved, we simply take $n_{\text {crit }}=1.5$ as a yardstick number and define the height corresponding to $n_{\text {crit }}$ as critical height $h_{\text {crit }}$ to quantify the onset point of the torus instability. We carried out a comprehensive investigation to evaluate to what extent the decay index affects solar eruptions, which has significant implications for space weather forecasting. We selected events from two-ribbon flares occurring during 2011-2015. The working assumption is that a magnetic flux rope is present in a classical two-ribbon flare, no matter if the rope pre-exists (e.g., Liu et al. 2010) or is newly formed (e.g., Wang et al. 2017). In the sections that follow, we elaborate on the procedure of calculation in Section 2 and give the statistical results and concluding remarks in Section 3.

\section{Observation and Analysis}

\subsection{Instruments}

This study mainly used data from the Helioseismic and Magnetic Imager (HMI; Scherrer et al. 2012) and the Atmospheric Imaging Assembly (AIA; Lemen et al. 2011) on board the Solar Dynamics Observatory (Pesnell et al. 2012), which was launched on 2010 February 11. HMI's hmi. sharp_cea data series provide disambiguated vector magnetograms that are deprojected to the heliographic coordinates with a Lambert (cylindrical equal area, CEA) projection method, at a cadence of $720 \mathrm{~s}$ and a pixel scale of $0^{\circ} .03$ (or $0.36 \mathrm{Mm}$; Bobra et al. 2014). Flares are monitored by the Geostationary Operational Environmental Satellite in soft X-ray (SXR) irradiance and by AIA's seven EUV imaging passbands $(94,131,171,193,211,304$, and $335 \AA$ ) and two UV imaging passbands (1600 and $1700 \AA)$ with a spatial resolution of 1 "! 5 and a temporal cadence of $12 \mathrm{~s}$ (24 s) for EUV (UV) passbands (Lemen et al. 2011). To obtain the context of CMEs, we examined coronagraph images obtained 
by Solar and Heliospheric Observatory (SOHO) and Solar Terrestrial Relations Observatory (STEREO).

\subsection{Selection and Category of Events}

Sixty two-ribbon flares of M- and X-class are selected in this study (Table 1) according to observations of UV flare ribbons in the chromosphere and of EUV post-flare arcades in the corona. The selection criterion is that the center of the source active region is located within $\sim 45^{\circ}$ from the solar disk center, so that the measurements of the photospheric magnetic field are relatively reliable. Flares are categorized as either "E" (eruptive) or " $\mathrm{C}$ " (confined) in Table 1. To determine whether a flare is associated with a CME, we collated coronagraph images obtained by $S O H O$ and STEREO, and EUV images obtained by AIA. The $S O H O$ LASCO CME catalog ${ }^{3}$ provides a benchmark reference for this purpose. Taking into account the timing and location of flares relative to CMEs as well as the CME speed and direction, we identified 35 eruptive and 25 confined flares (Table 1).

\subsection{Decay Index and Critical Height}

According to an analytical model of torus instability, a toroidal flux ring is unstable to lateral expansion if the external poloidal field $B_{\text {ex }}$ decreases rapidly with increasing height such that the decay index $n=-d \ln B_{\mathrm{ex}} / d \ln h$ exceeds $3 / 2$ (Kliem $\&$ Török 2006). Due to the difficulty in decoupling $B_{\mathrm{ex}}$ from the flux-rope field in either simulation or observation, a conventional practice is to approximate $B_{\mathrm{ex}}$ with a current-free potential field (e.g., Fan \& Gibson 2007; Török \& Kliem 2007; Liu 2008; Démoulin \& Aulanier 2010). In our study, the coronal potential field is extrapolated from the $B_{z}$ component of the vector magnetograms for active regions, using a Fourier transformation method (Alissandrakis 1981).

Hence, in our calculation, $n=-d \ln B_{t} / d \ln h$, where $B_{t}$ denotes the transverse component of the extrapolated potential field, i.e., $B_{t}=\sqrt{B_{x}^{2}+B_{y}^{2}}$. Precisely speaking, it is the external field component orthogonal to the axial current of the flux rope that creates the downward $\boldsymbol{J} \times \boldsymbol{B}$ force. $B_{t}$ often serves as a good approximation since potential field is almost orthogonal to PIL, along which a flux rope in equilibrium typically resides. One needs keep in mind that this approximation works better with less curved PILs. Here, we take as an example the confined flare on 2014 October 22 in NOAA AR 12192 (No. 52 in Table 1; see also Sun et al. 2015; Liu et al. 2016a) to demonstrate how the critical height $h_{\text {crit }}$ is calculated. Figure 1(a) shows a pre-flare photospheric $B_{z}$ map of AR 12192 at 13:48 UT prior to the onset of the flare, and Figure 1(b) shows the flare ribbons observed near the SXR peak at 14:28 UT in AIA $1600 \AA$ A. We sampled the segment of polarity inversion line (PIL) that is located in between the twoflare ribbons (referred to as "flaring PIL" hereafter) by clicking on it as uniformly as possible to get sufficient representative points (marked by crosses), and then calculated decay index $n$ at different heights at these selected points. In Figure 1(c), we plot $n$ as a function of $h$, which is averaged over the selected points, with the error bar indicating the standard deviation. We located the critical height corresponding to $n=1.5$ by linear interpolation between the discrete $n(h)$ points, which have a step of $0.36 \mathrm{Mm}$, and similarly we located the height at $n=1.5$

\footnotetext{
http://cdaw.gsfc.nasa.gov/CME_list/index.html
}

on the $n+\delta n$ and $n-\delta n$ profile, where $\delta n$ is the standard deviation at each $n(h)$ point, to get an uncertainty estimation of critical height. For this case, we obtained that $h_{\text {crit }}=$ $70.6_{-7.9}^{+8.4} \mathrm{Mm}$. As a comparison, Figures 1(d)-(f) show an eruptive flare taking place on 2012 March 14 (No. 12). The corresponding $h_{\text {crit }}=31.1_{-9.6}^{+7.8} \mathrm{Mm}$ is much smaller than the confined case.

To evaluate the complexity of magnetic field in active regions and its impact on $h_{\text {crit }}$, we calculated the centroids of positive and negative magnetic fluxes for each active region and their distance $d$. We propose that the magnetic field relevant to a flare of interest can be deemed the dipolar field (labeled " $\mathrm{D}$ " in Table 1) if the centroids of opposite polarities are located at two sides of, and their connection passes through, the flaring PIL (e.g., Figure 1(a)). In contrast, the magnetic field is deemed the multipolar field (labeled " $\mathrm{M}$ " in Table 1) if the connection of centroids fails to pass through (e.g., Figure 1(d)), or is almost parallel to, the flaring PIL. The latter category includes some cases in which the active region of interest cannot be clearly separated from a neighboring active region

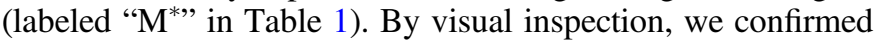
that this categorization gives a result consistent with the conventional view of dipolar and multipolar fields.

\section{Results}

The distribution of $h_{\text {crit }}$ for the sample of 60 two-ribbon flares is shown in the top panel of Figure 2. The total distribution of $h_{\text {crit }}$ peaks at the heights of 20-30 Mm, but for confined flares $h_{\text {crit }}$ significantly spreads to higher heights than eruptive flares. The average $h_{\text {crit }}$ is $36.3 \pm 17.4 \mathrm{Mm}$ for the 35 eruptive flares, and $53.6 \pm 21.3 \mathrm{Mm}$ for the 25 confined flares. $h_{\text {crit }}$ is highly correlated with the centroid distance $d$ of active regions (bottom panel of Figure 2). From the linear fittings using a least absolute deviation method (LADFIT in IDL), we obtained an empirical formula

$$
h_{\text {crit }} \simeq \frac{1}{2} d,
$$

which may serve as a rule of thumb for the scale of $h_{\text {crit. }}$ In comparison to numerical models, Kliem et al. (2014, Equation (15)) found that within the framework of the active-region model developed by Titov \& Démoulin (1999), $h_{\text {crit }} / L$ is slightly below unity, where $L$ is the half distance between two monopoles. This is derived for a freely expanding torus without being line-tied. In the numerical experiments with a line-tying surface (Török \& Kliem 2007, their Figures 2 and 3), one can also see that for bipolar configurations $h_{\text {crit }}$ increases when the distance between external sources increases and that Equation (1) approximately holds for each case (T. Török 2017 , private communication). On the other hand, $h_{\text {crit }}$ is found to be comparable to the horizontal distance between two subphotospheric monopoles in a series of numerical simulations imposing different photospheric flows and diffusive coefficients (Aulanier et al. 2010; Zuccarello et al. 2015, 2016). Generally speaking, $h_{\text {crit }}$ may be affected by various factors including, but not limited to, (1) functional form of the external field; (2) other external sources besides the dipole confining the flux rope; and (3) depths of the external sources below the surface. For example, in Török \& Kliem (2007), the monopoles 
Table 1

Flare List

\begin{tabular}{|c|c|c|c|c|c|c|c|c|c|}
\hline \multirow{2}{*}{ Number } & \multirow{2}{*}{$\begin{array}{l}\text { Date } \\
\text { YYYY MM DD }\end{array}$} & \multirow{2}{*}{$\begin{array}{l}\text { Time }^{\mathrm{a}} \\
\mathrm{hh} \mathrm{mm}\end{array}$} & \multicolumn{2}{|c|}{ Location } & \multirow{2}{*}{ Class } & \multirow{2}{*}{ Category $^{\mathrm{c}}$} & \multirow{2}{*}{ Profile $^{\mathrm{d}}$} & \multirow{2}{*}{ Configuration $^{\mathrm{e}}$} & \multirow{2}{*}{$\begin{array}{r}h_{\text {crit }} \\
(\mathrm{Mm})\end{array}$} \\
\hline & & & AR & Position $^{\mathrm{b}}$ & & & & & \\
\hline 1 & 2011 Mar 07 & 1430 & 11166 & N11E21 & M1.7 & $\mathrm{E}$ & I & $\mathrm{D}$ & $46.8_{-3.2}^{+3.2}$ \\
\hline 2 & 2011 Aug 02 & 0619 & 11261 & N17W22 & M1.4 & E & I & M & $22.2_{-6.0}^{+5.1}$ \\
\hline 3 & 2011 Oct 01 & 0959 & 11305 & N10W06 & M1.2 & $\mathrm{E}$ & I & M & $24.1_{-1.9}^{+2.5}$ \\
\hline 4 & 2011 Oct 02 & 0050 & 11305 & N09W12 & M3.9 & $\mathrm{E}$ & I & M & $19.4_{-2.3}^{+2.8}$ \\
\hline 5 & 2011 Dec 26 & 0227 & 11387 & S21W33 & M1.5 & $\mathrm{E}$ & S & M & $11.6_{-1.6}^{+3.9}$ \\
\hline 6 & 2011 Dec 26 & 2030 & 11387 & S21W44 & M2.3 & $\mathrm{E}$ & S & $\mathrm{M}$ & $9.3_{-1.9}^{+3.6}$ \\
\hline 7 & 2012 Jan 19 & 1605 & 11402 & N32E27 & M3.2 & $\mathrm{E}$ & I & $\mathbf{M}^{*}$ & $46.9_{-5.0}^{+6.0}$ \\
\hline 8 & 2012 Jan 23 & 0359 & 11402 & N28W21 & M8.7 & $\mathrm{E}$ & I & $\mathbf{M}^{*}$ & $46.0_{-6.1}^{+5.9}$ \\
\hline 9 & 2012 Mar 07 & 0024 & 11429 & N18E31 & X5.4 & $\mathrm{E}$ & I & $\mathrm{D}$ & $38.6_{-3.4}^{+2.9}$ \\
\hline 10 & 2012 Mar 07 & 0114 & 11429 & N15E26 & $\mathrm{X} 1.3$ & E & I & $\mathrm{D}$ & $39.1_{-8.1}^{+7.5}$ \\
\hline 11 & 2012 Mar 10 & 1744 & 11429 & N17W24 & M8.4 & E & I & $\mathrm{D}$ & $62.4_{-19.9}^{+10.4}$ \\
\hline 12 & 2012 Mar 14 & 1521 & 11432 & N13E05 & M2.8 & $\mathrm{E}$ & I & M & $31.1_{-9.6}^{+7.8}$ \\
\hline 13 & 2012 Mar 15 & 0752 & 11432 & N14W03 & M1.8 & $\mathrm{E}$ & I & $\mathrm{M}$ & $40.7_{-3.0}^{+2.4}$ \\
\hline 14 & 2012 Jun 06 & 2006 & 11494 & S19W05 & M2.1 & E & I & $\mathbf{M}^{*}$ & $20.0_{-1.4}^{+1.4}$ \\
\hline 15 & 2012 Jun 14 & 1435 & 11504 & S19E06 & M1.9 & E & I & $\mathrm{D}$ & $45.8_{-4.7}^{+4.4}$ \\
\hline 16 & 2012 Jul 05 & 1318 & 11515 & S16W43 & M1.2 & E & I & $\mathbf{M}^{*}$ & $68.9_{-5.2}^{+5.6}$ \\
\hline 17 & 2012 Jul 12 & 1649 & 11520 & S13W03 & $\mathrm{X} 1.4$ & E & I & $\mathbf{M}^{*}$ & $36.0_{-5.3}^{+5.0}$ \\
\hline 18 & 2013 May 16 & 2153 & 11748 & N11E40 & M1.3 & E & I & $\mathbf{M}^{*}$ & $21.9_{-2.4}^{+2.4}$ \\
\hline 19 & 2013 Aug 12 & 1041 & 11817 & S21E18 & M1.5 & E & I & $\mathbf{M}^{*}$ & $22.9_{-2.7}^{+3.4}$ \\
\hline 20 & 2013 Oct 13 & 0043 & 11865 & S22E17 & M1.7 & $\mathrm{E}$ & S & $\mathrm{M}$ & $15.3_{-4.3}^{+7.5}$ \\
\hline 21 & 2013 Oct 28 & 1153 & 11877 & S16W44 & M1.4 & $\mathrm{E}$ & I & $\mathrm{D}$ & $69.7_{-3.0}^{+3.4}$ \\
\hline 22 & 2014 Feb 12 & 0425 & 11974 & S12W02 & M3.7 & $\mathrm{E}$ & S & $\mathrm{M}$ & $64.8_{-14.0}^{+10.6}$ \\
\hline 23 & 2014 Dec 17 & 0110 & 12242 & S20E08 & M1.5 & E & I & $\mathbf{M}^{*}$ & $26.6_{-3.9}^{+4.3}$ \\
\hline 24 & 2014 Dec 17 & 0150 & 12241 & S11E33 & M1.1 & E & I & $\mathrm{M}$ & $15.9_{-3.6}^{+11.4}$ \\
\hline 25 & 2014 Dec 17 & 0451 & 12242 & S18E08 & M8.7 & $\mathrm{E}$ & I & $M^{*}$ & $27.7_{-5.1}^{+5.7}$ \\
\hline 26 & 2014 Dec 20 & 0028 & 12242 & S19W29 & $\mathrm{X} 1.8$ & $\mathrm{E}$ & I & $\mathrm{M}$ & $40.5_{-4.5}^{+4.3}$ \\
\hline 27 & $2014 \operatorname{Dec} 21$ & 1217 & 12241 & S13W25 & M1.0 & E & I & M & $51.7_{-21.5}^{+8.2}$ \\
\hline 28 & 2015 Mar 09 & 2353 & 12297 & S18E45 & M5.8 & $\mathrm{E}$ & I & M & $31.9_{-6.2}^{+5.8}$ \\
\hline 29 & 2015 Mar 15 & 2322 & 12297 & S19W32 & M1.2 & $\mathrm{E}$ & I & M & $20.1_{-5.3}^{+5.6}$ \\
\hline 30 & 2015 Mar 16 & 1058 & 12297 & S17W38 & M1.6 & E & S & M & $16.8_{-2.5}^{+1.9}$ \\
\hline 31 & 2015 Jun 21 & 0142 & 12371 & N12E13 & M2.0 & $\mathrm{E}$ & I & $\mathrm{D}$ & $46.3_{-12.9}^{+10.3}$ \\
\hline 32 & 2015 Jun 22 & 1823 & 12371 & N13W06 & M6.5 & $\mathrm{E}$ & I & $\mathrm{D}$ & $31.6_{-8.5}^{+12.3}$ \\
\hline 33 & 2015 Jun 25 & 0816 & 12371 & N12W40 & M7.9 & $\mathrm{E}$ & I & $\mathrm{D}$ & $56.6_{-7.4}^{+5.7}$ \\
\hline 34 & 2015 Nov 04 & 1352 & 12443 & N08W02 & M3.7 & $\mathrm{E}$ & I & $\mathrm{D}$ & $68.7_{-4.4}^{+3.9}$ \\
\hline 35 & 2015 Nov 09 & 1312 & 12449 & S13E39 & M3.9 & $\mathrm{E}$ & I & $\mathbf{M}^{*}$ & $35.3_{-4.5}^{+3.3}$ \\
\hline 36 & 2011 Mar 09 & 1107 & 11166 & N09W06 & M1.7 & $\mathrm{C}$ & I & $\mathrm{D}$ & $46.9_{-8.5}^{+7.1}$ \\
\hline 37 & 2011 Aug 03 & 0432 & 11261 & N17E12 & M1.7 & $\mathrm{C}$ & S & M & $16.9_{-1.6}^{+2.0}$ \\
\hline 38 & 2011 Nov 05 & 0335 & 11339 & N20E45 & M3.7 & $\mathrm{C}$ & I & M & $74.5_{-7.6}^{+8.2}$ \\
\hline 39 & 2011 Nov 05 & 1121 & 11339 & N19E41 & M1.1 & $\mathrm{C}$ & I & M & $74.9_{-8.3}^{+8.9}$ \\
\hline 40 & 2011 Nov 06 & 0103 & 11339 & N21E33 & M1.2 & $\mathrm{C}$ & I & $\mathrm{M}$ & $82.2_{-9.3}^{+9.7}$ \\
\hline 41 & 2011 Dec 31 & 1315 & 11389 & S25E46 & M2.4 & $\mathrm{C}$ & I & $\mathbf{M}^{*}$ & $61.4_{-2.3}^{+1.9}$ \\
\hline 42 & 2011 Dec 31 & 1626 & 11389 & $\mathrm{~S} 26 \mathrm{E} 42$ & M1.5 & $\mathrm{C}$ & I & $\mathbf{M}^{*}$ & $62.5_{-3.4}^{+2.5}$ \\
\hline 43 & 2012 Mar 06 & 1241 & 11429 & N18E36 & M2.1 & $\mathrm{C}$ & I & $\mathrm{D}$ & $37.9_{-7.6}^{+5.1}$ \\
\hline 44 & 2012 Apr 27 & 0824 & 11466 & N11W30 & M1.0 & $\mathrm{C}$ & I & $\mathbf{M}^{*}$ & $27.2_{-2.0}^{+2.1}$ \\
\hline 45 & 2012 May 09 & 1408 & 11476 & N06E22 & M1.8 & $\mathrm{C}$ & I & M & $33.6_{-3.8}^{+4.1}$ \\
\hline 46 & 2012 Jul 10 & 0514 & 11520 & S16E35 & M1.7 & $\mathrm{C}$ & I & $\mathbf{M}^{*}$ & $38.4_{-4.2}^{+3.6}$ \\
\hline 47 & 2013 Nov 01 & 1953 & 11884 & S12E01 & M6.3 & $\mathrm{C}$ & S & $\mathbf{M}^{*}$ & $71.2_{-7.2}^{+6.7}$ \\
\hline 48 & 2014 Feb 04 & 0400 & 11967 & S14W07 & M5.2 & $\mathrm{C}$ & $\mathrm{S}$ & $\mathrm{M}$ & $28.4_{-6.6}^{+7.5}$ \\
\hline 49 & 2014 Feb 06 & 2305 & 11967 & S15W48 & M1.5 & $\mathrm{C}$ & $\mathrm{S}$ & M & $19.4_{-3.3}^{+5.4}$ \\
\hline 50 & 2014 Oct 20 & 0911 & 12192 & S16E42 & M3.9 & $\mathrm{C}$ & I & $\mathrm{D}$ & $78.7_{-14.1}^{+11.8}$ \\
\hline 51 & 2014 Oct 20 & 1637 & 12192 & S14E39 & M4.5 & $\mathrm{C}$ & I & $\mathrm{D}$ & $82.1_{-15.2}^{+12.6}$ \\
\hline 52 & 2014 Oct 22 & 1428 & 12192 & S14E13 & X1.6 & $\mathrm{C}$ & I & $\mathrm{D}$ & $70.6_{-7.9}^{+8.4}$ \\
\hline 53 & 2014 Oct 24 & 2141 & 12192 & S22W 21 & $\mathrm{X} 3.1$ & $\mathrm{C}$ & I & $\mathrm{D}$ & $84.0_{-9.9}^{+10.5}$ \\
\hline 54 & 2014 Dec 01 & 0641 & 12222 & S22E17 & M1.8 & $\mathrm{C}$ & I & $\mathbf{M}^{*}$ & $57.1_{-1.4}^{+1.6}$ \\
\hline 55 & 2014 Dec 17 & 1901 & 12241 & S10E23 & M1.4 & $\mathrm{C}$ & I & $\mathrm{M}$ & $48.6_{-6.1}^{+6.6}$ \\
\hline 56 & 2014 Dec 18 & 2158 & 12241 & S11E10 & M6.9 & $\mathrm{C}$ & I & M & $54.7_{-4.9}^{+5.1}$ \\
\hline 57 & 2014 Dec 19 & 0944 & 12242 & S19W27 & M1.3 & $\mathrm{C}$ & $\mathrm{I}$ & $\mathbf{M}^{*}$ & $48.7_{-9.5}^{+17.1}$ \\
\hline
\end{tabular}


Table 1

(Continued)

\begin{tabular}{|c|c|c|c|c|c|c|c|c|c|}
\hline \multirow{2}{*}{ Number } & \multirow{2}{*}{$\begin{array}{l}\text { Date } \\
\text { YYYY MM DD }\end{array}$} & \multirow{2}{*}{$\begin{array}{l}\text { Time }^{\mathrm{a}} \\
\text { hh mm }\end{array}$} & \multicolumn{2}{|c|}{ Location } & \multirow{2}{*}{ Class } & \multirow{2}{*}{ Category $^{c}$} & \multirow{2}{*}{ Profile $^{\mathrm{d}}$} & \multirow{2}{*}{ Configuration $^{\mathrm{e}}$} & \multirow{2}{*}{$\begin{array}{r}h_{\text {crit }} \\
(\mathrm{Mm})\end{array}$} \\
\hline & & & $\mathrm{AR}$ & Position $^{b}$ & & & & & \\
\hline 58 & 2015 Jan 03 & 0947 & 12253 & S05E16 & M1.1 & C & I & D & $58.9_{-2.5}^{+1.9}$ \\
\hline 59 & 2015 Jan 04 & 1536 & 12253 & S05E01 & M1.3 & $\mathrm{C}$ & I & $\mathrm{D}$ & $62.5_{-0.8}^{+0.8}$ \\
\hline 60 & 2015 Mar 11 & 1851 & 12297 & S15E18 & M1.0 & $\mathrm{C}$ & I & M & $19.6_{-6.4}^{+5.2}$ \\
\hline
\end{tabular}

Notes.

a GOES $1-8 \AA$ A peak time.

${ }^{\mathrm{b}}$ Flare position provided by GOES.

c "E" for eruptive flares, "C" for confined flares.

d "I" for monotonous increasing of $n$ as a function of $h$, "S" for a saddle-like $n(h)$ profile.

e " $D$ " for a dipolar magnetic field, " $M$ " for a multipolar field, and " $M$ "' indicates that the active region of interest is too close to be separated from a neighboring active region.
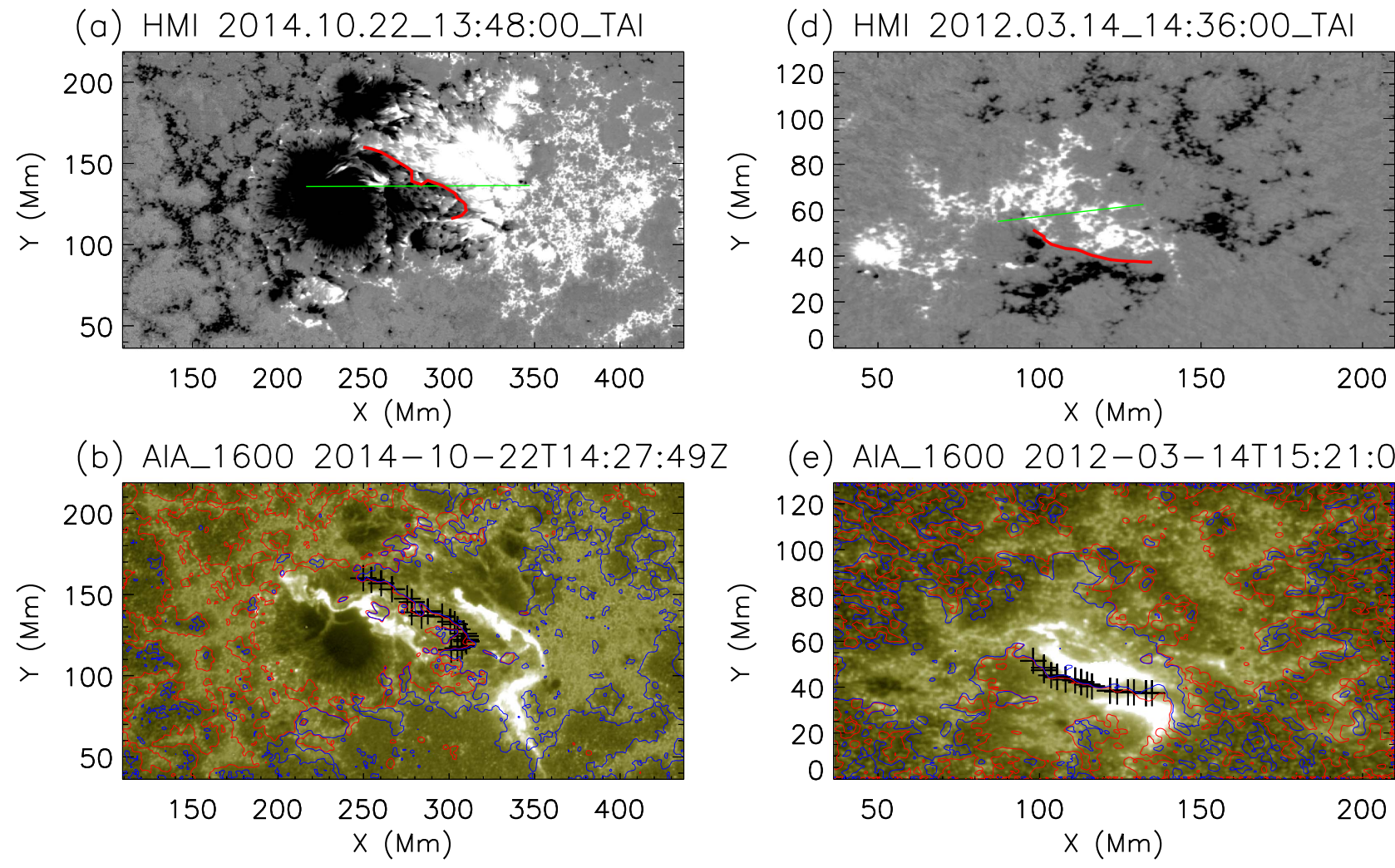

(e) AIA_1600 2012-03-14T15:21:07Z
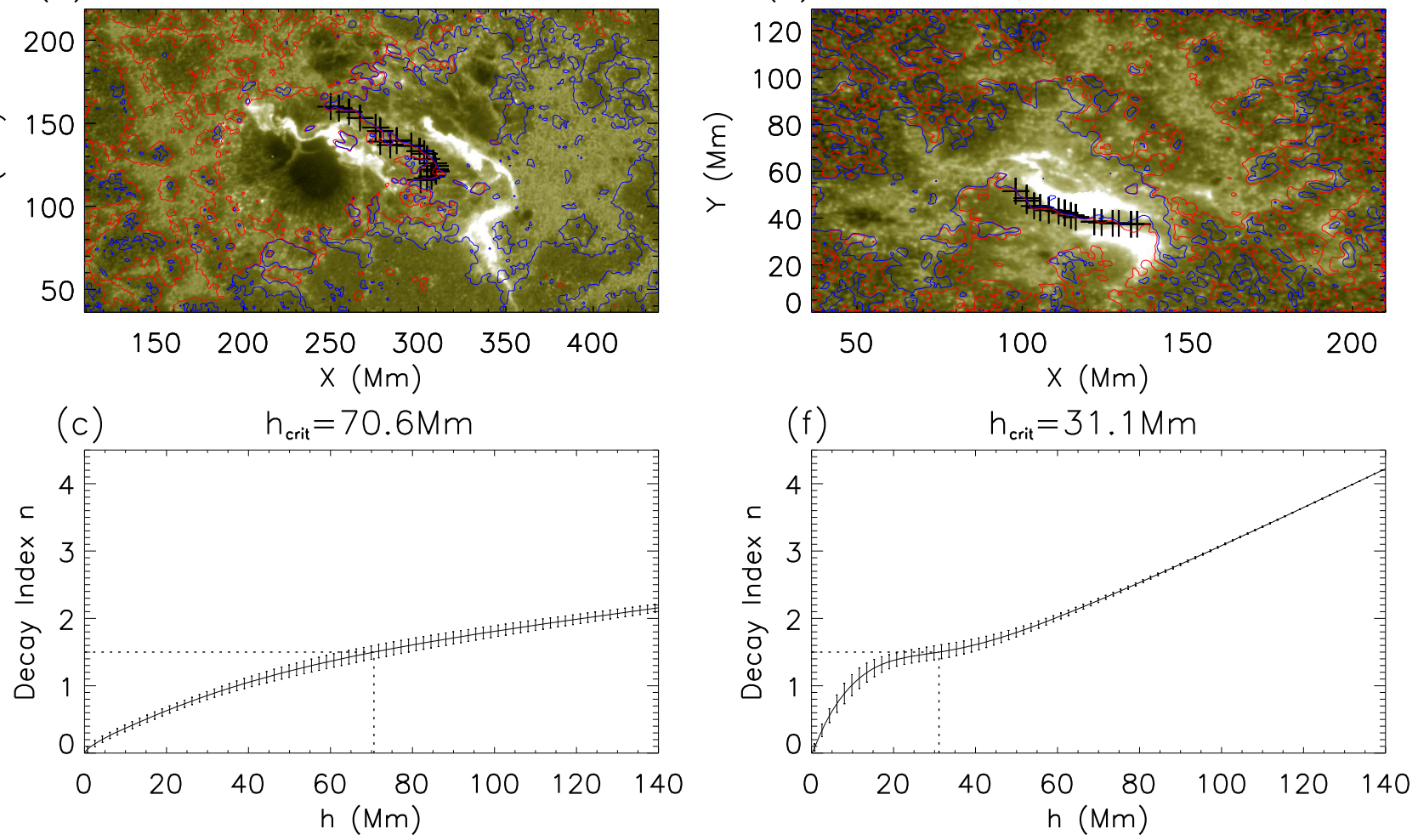

Figure 1. Derivation of the decay index profile for two exemplary events, a confined flare (No. 52) on the left and an eruptive flare (No. 12) on the right. ((a) and (d)) HMI $B_{z}$ map. The red line denotes the flaring PIL and the green line connects the centroids of opposite polarities. ((b) and (e)) The AIA $1600 \AA$ image overlaid by the $B_{z}$ contour (50 and $10 \mathrm{G}$ ), with red (blue) colors indicating negative (positive) polarity. The sign "+" denotes the points selected along the flaring PIL. ((c) and (f)) The decay index $n$ as a function of the height $h$ above the surface in units of Mm. Dotted lines indicate where $n_{\text {crit }}$ and $h_{\text {crit }}$ are taken. 

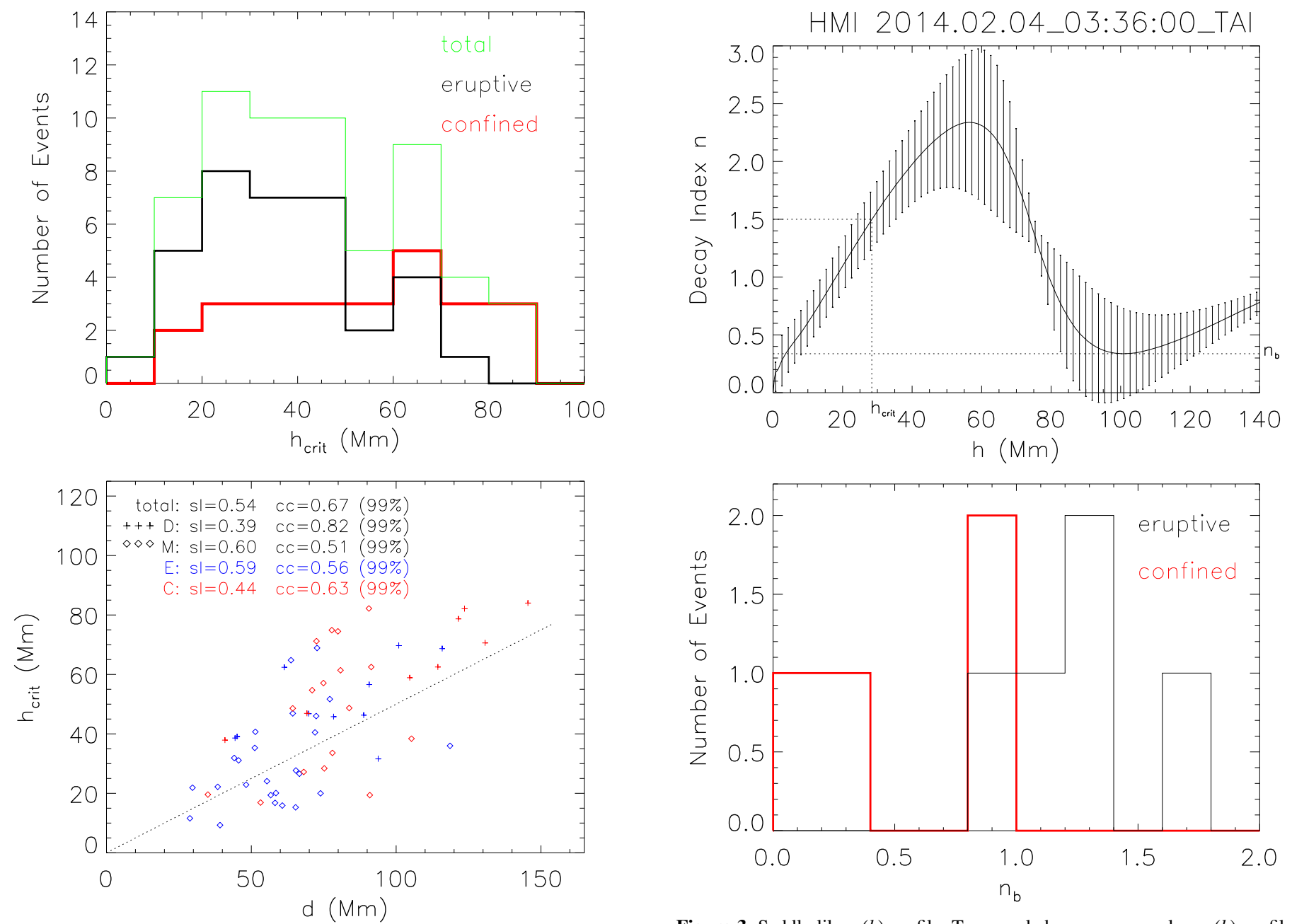

Figure 2. Distribution of $h_{\text {crit }}$ (top) and its relation to the centroid distance $d$ of active regions (bottom). In the bottom panel, the plus and diamond symbols denote dipolar (D) and multipolar (M) magnetic field, respectively. Eruptive (E) and confined (C) events are shown in blue and red, respectively. "sl" indicates the slope given by linear fitting and "cc" the correlation coefficient with the confidence interval denoted in the brackets. $h_{\text {crit }}=\frac{1}{2} d$ is marked by the dotted line.

are very close to the surface, as compared to the significant depths set in Aulanier et al. (2010).

Two distinct types of $n(h)$ profiles emerge in this investigation, similar to a much smaller sample of nine flares studied by Cheng et al. (2011): (1) $n$ increases monotonically as the height increases in 30 of 35 (86\%) eruptive flares and in 21 of 25 (84\%) confined flares; and (2) the rest of the $n(h)$ profiles are saddle-like, exhibiting a local minimum at a height higher than $h_{\text {crit }}$ (e.g., top panel of Figure 3). The saddle-like profile provides a potential to confine an eruptive structure if the local minimum $n_{b}$ at the bottom of the saddle is significantly below $n_{\text {crit }}$ and the eruption has not developed a large enough disturbance when the eruptive structure reaches the height of $n_{b}$. For example, the deep saddle bottom at higher altitudes than $h_{\text {crit }}$ may help confine the eruption on 2014 February 4 (top panel of Figure 3). For the nine flares exhibiting a saddle-like $n(h)$ profile, including five eruptive and four confined flares, the distribution of $n_{b}$ is given in the bottom panel Figure 3. One can see that $n_{b}$ of the five eruptive flares (black) is generally larger than that of the four confined flares (red). In relation to the field configuration, an outstanding characteristic for saddle-like profiles is that all nine

Figure 3. Saddle-like $n(h)$ profile. Top panel shows an exemplary $n(h)$ profile from the confined flare on 2014 February 4 (No. 48 in Table 1). $n_{b}$ and $h_{\text {crit }}$ are marked. Bottom panel shows the distribution of $n_{b}$ for five eruptive (black) and four confined (red) flares.

Table 2

Flare Statistics

\begin{tabular}{lrrrr}
\hline \hline & \multicolumn{1}{c}{ E } & \multicolumn{1}{c}{ C } & \multicolumn{1}{c}{ I } & \multicolumn{1}{c}{ S } \\
\hline $\mathrm{D}$ & 10 & 8 & 18 & 0 \\
$\mathrm{M}\left(\mathrm{M}^{*}\right)$ & $25(10)$ & $17(7)$ & $33(16)$ & $9(1)$ \\
\hline
\end{tabular}

Note. Number of different types of flares (E and C) and of $n(h)$ profiles (I and S) in relation to magnetic field configuration ( $\mathrm{D}$ and $\mathrm{M})$ of active regions. The same notations are adopted here as in Table 1.

events originate from the multipolar magnetic field (Table 2). However, it is not clear exactly what kind of photospheric flux distribution would yield the saddle shape because, on the one hand, the relevant magnetic field is highly complex; on the other hand, the majority cases of monotonously growing $n(h)$ also originates from the multipolar field (Table 2). This will be considered in a future investigation.

To conclude, this investigation confirms that the decay index profile of the background field plays an important role in deciding whether a two-ribbon flare would lead up to a CME. Moreover, the saddle-like profile present in some active regions may provide an additional confinement effect on eruptions. 
These results indicate the possibility that some two-ribbon flares might be innately incapable of producing CMEs.

The authors thank the anonymous reviewer for constructive comments that helped greatly improve this Letter. D.W. acknowledges the support by Natural Science Foundation of Anhui province education department (KJ2016JD18, KJ2017A493). R.L. acknowledges the support by NSFC 41474151 and the Thousand Young Talents Program of China, and thanks T. Török for helpful comments. Y.W. acknowledges the support from NSFC 41131065 and 41574165. This work was also supported by NSFC 41421063, CAS Key Research Program KZZD-EW-01-4, and the fundamental research funds for the central universities.

\section{References}

Alissandrakis, C. E. 1981, A\&A, 100, 197

Aulanier, G., Török, T., Démoulin, P., \& DeLuca, E. E. 2010, ApJ, 708, 314

Bobra, M. G., Sun, X., Hoeksema, J. T., et al. 2014, SoPh, 289, 3549

Cheng, X., Zhang, J., Ding, M., Guo, Y., \& Su, J. 2011, ApJ, 732, 87

Démoulin, P., \& Aulanier, G. 2010, ApJ, 718, 1388

Fan, Y., \& Gibson, S. E. 2007, ApJ, 668, 1232

Harrison, R. 2003, AdSpR, 32, 2425

Kliem, B., Lin, J., Forbes, T. G., Priest, E. R., \& Török, T. 2014, ApJ, 789, 46

Kliem, B., Su, Y. N., van Ballegooijen, A. A., \& DeLuca, E. E. 2013, ApJ, 779, 129 Kliem, B., \& Török, T. 2006, PhRvL, 96, 255002
Lemen, J. R., Akin, D. J., Boerner, P. F., et al. 2011, The Solar Dynamics Observatory (Berlin: Springer), 17

Liu, C., Deng, N., Liu, R., et al. 2015, ApJL, 812, L19

Liu, L., Wang, Y., Wang, J., et al. 2016a, ApJ, 826, 119

Liu, R., Chen, J., Wang, Y., \& Liu, K. 2016b, NatSR, 6, 34021

Liu, R., Liu, C., Wang, S., Deng, N., \& Wang, H. 2010, ApJL, 725, L84

Liu, R., Kliem, B., Titov, V. S., et al. 2016, ApJ, 818, 148

Liu, Y. 2008, ApJL, 679, L151

Myers, C. E., Yamada, M., Ji, H., et al. 2015, Natur, 528, 526

Myers, C. E., Yamada, M., Ji, H., et al. 2016, PhPl, 23, 112102

Myers, C. E., Yamada, M., Ji, H., et al. 2017, PPCF, 59, 014048

Pesnell, W. D., Thompson, B. J., \& Chamberlin, P. C. 2012, SoPh, 275, 3

Priest, E., \& Forbes, T. 2002, A\&ARv, 10, 313

Scherrer, P. H., Schou, J., Bush, R., et al. 2012, SoPh, 275, 207

Shibata, K. 1998, Ap\&SS, 264, 129

Sun, X., Bobra, M. G., Hoeksema, J. T., et al. 2015, ApJL, 804, L28

Titov, V., \& Démoulin, P. 1999, A\&A, 351, 707

Török, T., \& Kliem, B. 2005, ApJL, 630, L97

Török, T., \& Kliem, B. 2007, AN, 328, 743

van Tend, W., \& Kuperus, M. 1978, SoPh, 59, 115

Wang, W., Liu, R., Wang, Y., et al. 2017, NatCo, submitted

Wang, Y., \& Zhang, J. 2007, ApJ, 665, 1428

Xu, Y., Liu, C., Jing, J., \& Wang, H. 2012, ApJ, 761, 52

Yashiro, S., Gopalswamy, N., Akiyama, S., Michalek, G., \& Howard, R. A. 2005, JGRA, 110, A12S05

Zhang, J., Dere, K., Howard, R., Kundu, M., \& White, S. 2001, ApJ, 559,452

Zhang, J., Dere, K., Howard, R., \& Vourlidas, A. 2004, ApJ, 604, 420

Zuccarello, F., Aulanier, G., \& Gilchrist, S. 2016, ApJL, 821, L23

Zuccarello, F. P., Aulanier, G., \& Gilchrist, S. A. 2015, ApJ, 814, 126

Zuccarello, F. P., Seaton, D. B., Mierla, M., et al. 2014, ApJ, 785, 88 